\title{
Acute Effects of Different Types of Resistance Training on Cardiac Autonomic Modulation in COPD
}

\author{
Franciele M Vanderlei PhD, Fernando Zandonadi MD, Fabiano Francisco de Lima MSc, \\ Bruna S A Silva MSc, Ana Paula C F Freire MSc, Dionei Ramos PhD, and \\ Ercy Mara C Ramos PhD
}

\begin{abstract}
BACKGROUND: An exercise modality that has been gaining significant importance in the rehabilitation of subjects with COPD is resistance training. When considering that patients with COPD present alterations in autonomic cardiac modulation caused by the disease itself, it is necessary to investigate the behavior of the autonomic nervous system in relation to this type of exercise. Thus, the objective of this study was to compare the acute effects of resistance training with elastic tubes, elastic bands, and conventional weightlifitng on the behavior of cardiac autonomic modulation in post-exercise recovery in subjects with COPD. METHODS: Thirty-four subjects with COPD performed an single session of resistance training divided according to the therapeutic resource used: elastic tubes $(n=10)$, elastic bands $(n=11)$, and conventional bodybuilding $(n=13)$. For analysis of cardiac autonomic modulation, the heart rate was obtained beat to beat at rest and immediately after the end of the session for $60 \mathrm{~min}$ in a seated position. Heart rate variability indices were obtained in the time and frequency domains. RESULTS: The 3 therapeutic resource types used in the single session of resistance training promoted changes in heart rate variability linear indices in the time and frequency domains; however, post-exercise recovery time was similar for all protocols performed. CONCLUSIONS: After single resistance training the elastic tubes group presented a minimum alteration in the post-exercise recovery of cardiac autonomic modulation in the subjects with COPD; however, at 5 min after exercising, the subjects with COPD had already recovered. Therefore, if the purpose of the training is to restore autonomic cardiac modulation, the use of elastic tubes is suggested, when considering their low cost and versatility. Key words: heart rate variability; autonomic nervous system; physical exercise; resistance training; COPD; rehabilitation; elastic tubes. [Respir Care 2018;63(8):1050-1059. (C) 2018 Daedalus Enterprises]
\end{abstract}

\section{Introduction}

COPD is a preventable, treatable, but not fully reversible respiratory disease characterized by chronic airway obstruction. ${ }^{1,2}$ One of the main ways of treating COPD with the goal of improving muscle condition is pulmonary rehabilitation. ${ }^{3,4}$ Among the therapeutic modalities used in the rehabilitation of patients with COPD, resistance train-

\footnotetext{
The authors are affiliated with the Department of Physical Therapy, Universidade Estadual Paulista - FCT/UNESP, Presidente Prudente, Sao Paulo, Brazil.
}

The authors have disclosed no conflicts of interest.

Drs Vanderlei and Zandonadi contributed equally to this work. ing has been gaining prominence. ${ }^{5}$ Researchers in the field are currently striving to detect possible treatment alternatives that offer greater versatility and better cost/benefit, ${ }^{6}$ and, within this perspective, elastic tubes ${ }^{7,8}$ and bands 9,10 have become an important therapeutic possibility.

It should be noted that the updated training methods for patients with COPD are considered to be autonomous modifications that occur during implementation of the training

\footnotetext{
Correspondence: Franciele M Vanderlei PhD, Ciências e Tecnologia FCT/UNESP, Departamento de Fisioterapia, Rua Roberto Simonsen 305, Presidente Prudente - SP CEP 19060-900, Brazil. E-mail: franmvanderlei@gmail.com.
}

DOI: $10.4187 /$ respcare .05898 


\section{Post-Exercise Recovery from Heart Rate Variability in COPD}

period and a recovery period. ${ }^{11}$ Thus, heart rate variability has been used as a noninvasive measurement tool to verify the effects of physical exercise on the autonomic nervous system, both in physiological and pathological conditions. ${ }^{12,13}$

It is known that patients with COPD present with alterations in cardiac autonomic modulation that lead to an increase in resting heart rate and a reduction in heart rate variability, which indicates specifically that their evaluation plays a role in the morbidity and mortality of these patients. ${ }^{14,15}$ However, in patients with COPD, the influence of different types of resources used in resistance training on cardiac autonomic modulation and post-exercise recovery has been poorly explored. Thus, when considering physical exercise as a stressor agent of of the body withdrawing the body from a condition of homeostasis and producing alterations from metabolic to autonomic, ${ }^{16,17}$ it is important to study modifications in cardiac autonomic modulation of patients with COPD, which already presents heart rate variability changes caused by the disease itself. ${ }^{15,16}$

In addition, it is worth reiterating that, among the different types of physical exercises proposed for this population, resistance training has been gaining followers due to its potential benefits. Therefore, it is important to study which type of resistance exercise provides better recovery of autonomic cardiac modulation to ensure that the patient can safely receive a new stimulus, in addition to ensuring patient safety at the end of pulmonary rehabilitation and when prescribing home exercises.

The objective of the present study was to compare the acute effects of resistance training with elastic tubes, elastic bands, and conventional bodybuilding on the behavior of cardiac autonomic modulation in post-exercise recovery in subjects with COPD. It was hypothesized that elastic resistance training would provide greater modification in cardiac autonomic modulation in COPD by generating greater muscle demand because the elastic resistance offers a linear increase in tension from the beginning to the end of the contraction during the movement. ${ }^{7,8}$

\section{Method}

\section{Population}

For this study, data from subjects of both sexes diagnosed with COPD were analyzed according to the Global Initiative for Chronic Obstructive Lung Disease, ${ }^{2}$ clinically stable without exacerbations or changes in medications for at least $30 \mathrm{~d}$ and without participating in any type of physical activity and/or pulmonary rehabilitation in the previous 6 months.

The study did not include volunteers who presented any of the following characteristics: current smokers, the use

\section{QUICK LOOK}

\section{Current knowledge}

It is known that patients with COPD present with alterations in cardiac autonomic modulation. Various physical exercises have been proposed in this population to improve health status, including resistance training with elastic resistance. However, identifying the effects of these therapeutic modalities on autonomic cardiac modulation is necessary to ensure safe prescription for the patient.

\section{What this paper contributes to our knowledge}

Subjects with COPD using elastic tubes for single resistance training had a minimum alteration in post-exercise recovery of cardiac autonomic modulation, and had already recovered at $5 \mathrm{~min}$ post exercise. Therefore, if the purpose of the training is to restore autonomic cardiac modulation, the use of elastic tubes is suggested because of their low cost and versatility.

of drugs that influence autonomic activity of the heart, people with alcoholism, people with known metabolic and/or endocrine disorders, home oxygen therapy, pathological conditions that impede the practice of physical training, unstable COPD, and participation in any other systematized exercise programs. Nonattendance in the training protocol for personal reasons, respiratory exacerbations during the training process, and time series of RR intervals that did not present $>95 \%$ sinus beats were considered exclusion criteria for the study.

A total of 81 subjects without clinical and respiratory exacerbations with COPD were eligible for the study, and 48 subjects were included. The subjects were randomly allocated to the following 3 groups by drawing lots: conventional training $(n=16)$, elastic tube training $(n=18)$, and elastic band training $(n=14)$. Anonymity was preserved by allocating a number to each subject before the draw. Random assignment was achieved by an independent person who drew the sealed numbers before the start of the intervention. The participants were blinded to the study hypotheses. During the analyses, some volunteers were excluded due to errors in heart rate variability capture, so the final composition of the groups was as follows: conventional training $(n=13$; degree $\mathrm{II}=5$, degree $\mathrm{III}=6$, degree IV $=2)$; elastic tube training $(n=10$; degree $\mathrm{II}=6$, degree III $=4)$; and elastic band training $(n=11$; degree II $=3$, degree III $=6$, degree IV $=2$ ). The sample loss flowchart is presented in Figure 1.

The volunteers were informed of the objectives and procedures of this study and, after agreeing, signed an informed consent form. All the procedures used were ap- 


\section{Post-Exercise Recovery from Heart Rate Variability in COPD}

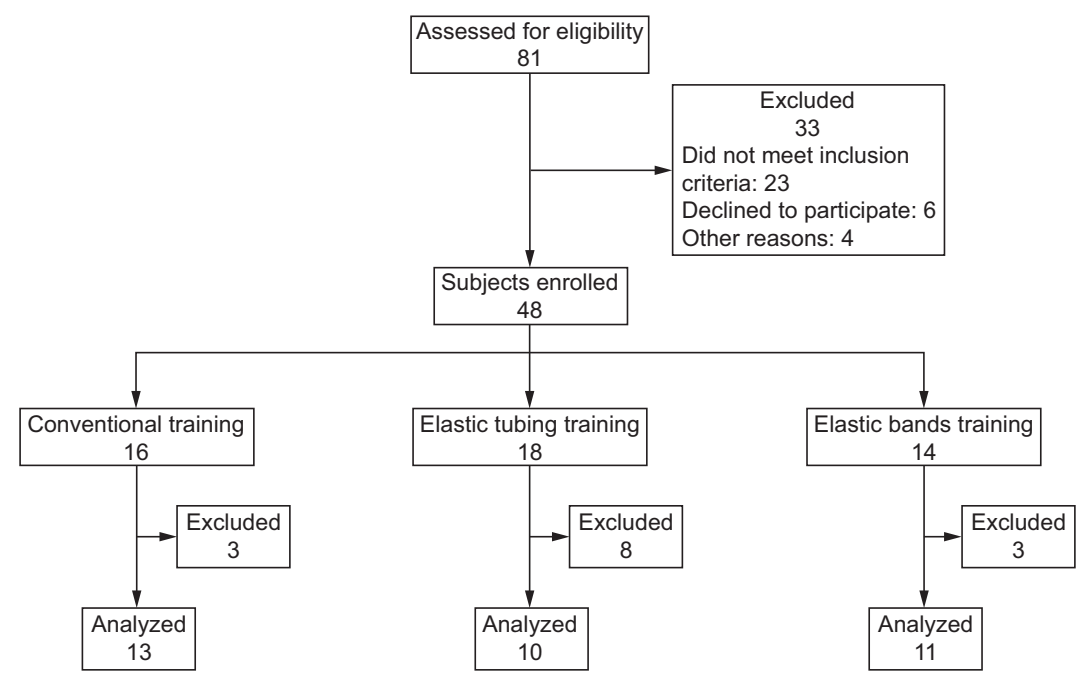

Fig. 1. Flow chart. Subjects excluded from final analysis due to mistakes in heart rate variability capture.

proved by the research ethics committee of the Universidade Estadual Paulista under protocol number CAAE: 46065315.7.0000.5402 and complied with Resolution 196/96 of the National Health Council. The clinical trial was registered in the Brazilian Registry of Clinical Trials under protocol number RBR-6v9sjj.

\section{Study Design}

This was a prospective experimental study started in March 2015 and completed in July 2016. Data collection took place in the Center for Studies and Assistance in Physiotherapy and Rehabilitation of the Faculty of Science and Technology - FCT/UNESP, in the morning to avoid variation in circadian cycle. To reduce the anxiety of the volunteers during the protocols, only a small number of people circulated through the site. Before performing the protocol, the volunteers were instructed to eat a light meal and dress in appropriate and comfortable clothes for the physical effort. In addition, they were instructed not to ingest coffee, black tea, or chocolate. After being instructed on these guidelines, the volunteers gave their written consent and were submitted to the procedures described below.

The collection was performed to verify the autonomic cardiac modulation recovery against the acute performance of 3 different resistance training protocols. On reaching the collection site, the following variables were verified: age, weight, stature, systolic and diastolic blood pressure, oxygen saturation, subjective effort scale (Borg), and breathing frequency. Information on the volunteer was identified through the medical history with questions regarding any severe cardiovascular-, neuromuscular-, skeletal-, or lung-associated diseases, and any history of diseases and current clinical state (medications currently prescribed, and the presence of diabetes, arterial hypertension, and smoking risk factors). Anthropometry was performed to characterize the population and to investigate the presence of excess weight and obesity. ${ }^{18}$ Spirometry was performed to confirm the diagnosis of COPD and classify the severity of the air flow. ${ }^{19}$

A heart rate monitor (model RS800cx; Polar Electro Oy, Kempele, Finland) was then placed on the volunteers to record the pulse rate throughout the protocol, while they remained at rest in a sitting position for $20 \mathrm{~min}$. The participants were instructed not to talk, make sudden movements, or sleep during this period. At the end of the rest period, the volunteers performed resistance training according to their previously randomized group: conventional, elastic bands, and elastic tubes. Immediately after the end of the training, the volunteers were again instructed to remain in a seated position for $60 \mathrm{~min}$, which thus initiated the heart rate variability recovery period. The heart rate variability analysis protocol was established based on studies that analyzed post-exercise recovery. ${ }^{20,21}$

\section{Training Protocols}

The exercise session lasted $60 \mathrm{~min}$, and, at the beginning and end of the session, the vital signs were verified and overall stretches were performed, such as of the trunk musculature and the upper and lower limbs. For the 3 protocols, exercises were performed for shoulder flexion, elbow flexion, shoulder abduction, knee extension, and knee flexion, with 2 sets of 15 repetitions and a passive rest interval of 1-2 min between sets. For the resistance training, the load used for each subject was defined according to the maximum repetition test.

For the maximum repetitions test, the subject was required to perform 15 maximum repetitions; the number of 


\section{Post-Exercise Recovery from Heart Rate Variability in COPD}

repetitions used for the initial test. Therefore, the test was valid when performed with a load (tubes, bands, or machines) that allowed the execution of 15 maximum repetitions. The test was considered invalid if the subject did not complete the range of motion and/or compensations. Both the test and the training were tolerated to execute up to 2 maximum repetitions. The participants performed up to 3 trials with a 2-min interval between attempts, and verbal stimulation was given to the subjects throughout the trials.

For the conventional resistance training, bodybuilding equipment (Ipiranga, São Paulo, Brazil) was used, being a simple pulley for the upper limbs and a flexor and extensor chair for the lower limbs. Resistance training with elastic tubes was performed by using Lemgruber latex elastic tubes, of different diameters and thicknesses, in addition to metal rings, bundles (plastic cable) for fixing the elastic tubes to the rings, and a specific chair with hooks for fixing the tubes and handles for the upper limbs. For the resistance training with elastic bands, a variety of Theraband elastic bands (Theraband ${ }^{\circledR}$, Akron, Ohio) were used (yellow [1.3-kg resistance with $100 \%$ elongation percentage], red [1.8 kg], green [2.3 kg], blue [3.2 kg], and black [4.4 kg]). Determination of the length of the elastic tubes and elastic bands was performed according to the distance from the upper or lower limb of each subject to the hook (fixed point) on the chair. Thus, the length differed from one subject to another according to the recommendations proposed by Ramos et al. ${ }^{8}$

Three senior physical therapists who specialized in pulmonary rehabilitation and/or musculoskeletal rehabilitation (FFL, BSAS and APCFF) and one physical therapist (FZ) were responsible for the assessment and management of the exercise sessions in the three intervention groups. The physical therapists provided close supervision during the sessions for all training protocols. The researcher who performed the data analyses was blinded to group allocation throughout the study.

\section{Analysis of Heart Rate Variability}

Heart rate variability analysis was performed from the series of RR intervals captured by using the Polar Electro Oy and linear methods, analyzed in the time and frequency domains. ${ }^{13}$ For this analysis, the time series of RR intervals was initially subjected to digital filtering by using Polar Pro Trainer software, complemented by manual filtering for elimination of premature ectopic beats and artifacts. Only series with $>95 \%$ sinus beats were included in the study. ${ }^{22}$ Visual analysis of the time series was performed to verify the absence of ectopic artifacts or beats that could interfere with the heart rate variability analysis.

For the analysis of heart rate variability in the time domain, the root-mean square of differences between ad- jacent normal RR intervals in a time interval (rMSSD) and standard deviation of all normal RR intervals (SDNN) ${ }^{13}$ were used which reflect respectively vagal modulation and global variability. In the frequency domain, spectral components of low frequency (LF) (global variability) and high frequency (HF) (vagal modulation), in $\mathrm{ms}^{2}$ and normalized units (nu), and the relationship between them (LF/ HF) were used..$^{13}$ The frequency bands used for each component were $\mathrm{LF}=0.04-0.15 \mathrm{~Hz}$ and $\mathrm{HF}=0.15-0.40 \mathrm{~Hz}$. The spectral analysis was calculated by using fast Fourier transform. ${ }^{13}$ All heart rate variability indices were processed by using Kubios HRV software (Biosignal Analysis and Medical Imaging Group, Kuopio, Finland), version 2.0.23

The series of RR intervals were analyzed at the following moments: M1 (5 min at rest), M2 (5 min initial recovery), M3 (5th-10th min of recovery), M4 (15th-20th min of recovery), M5 (25th-30th min of recovery), M6 (40th45th min of recovery), and M7 (55th-60th min of recovery), which contained 256 consecutive RR intervals in these intervals. ${ }^{12}$

\section{Statistical Analysis}

For analysis of the data of the population profile, the descriptive statistical method was used, and the results are presented as mean $\pm \mathrm{SD}$, median, minimum and maximum numbers, and CI. The normality of the data was evaluated by using the Shapiro-Wilks test. For comparison of the characteristics of the sample, one-way analysis of variance (ANOVA) was used with the Tukey honest significant difference test (age, weight, and body mass index) and the Kruskal-Wallis test with Dunn test (height).

The comparisons of heart rate variability indices among the protocols (conventional vs elastic tube vs elastic band) and moments (rest vs recovery times) were performed by using the technique of ANOVA for models of repeated measurements in the 2-factor scheme (mixed ANOVA). Repeated measurement data were checked for breach of sphericity by using the Mauchly test, and the GreenhouseGeisser correction was used when sphericity was violated.

For analysis of the moments (rest vs recovery times), the Bonferroni test was used for parametric or the Dunnett test for non-parametric distribution and the analysis of the different moments between the groups was carried out by using one-way ANOVA or the Kruskal-Wallis test. Statistical significance was set at $5 \%$ for all analyzes.

The sample was calculated based on the study by Santos et $\mathrm{al}^{24}$ in which an index of instantaneous recording of beat-to-beat variability (SD1) (reflecting vagal modulation) was selected. The difference to be detected was 7.97 $\mathrm{ms}$ and the SD was $6.45 \mathrm{~ms}$. The significance level for the sample calculation was 5\%, with a test power of $80 \%$ and 


\section{Post-Exercise Recovery from Heart Rate Variability in COPD}

Table 1. Age, Weight, BMI, and Height of the 3 Groups Analyzed

\begin{tabular}{lccc}
\hline \hline \multicolumn{1}{c}{ Variables } & Elastic Tube Group & Elastic Band Group & Conventional Group \\
\hline Age, mean $\pm \mathrm{SD} \mathrm{y*}$ & $72.16 \pm 8.36$ & $66.15 \pm 7.91$ & $64.84 \pm 12.02$ \\
Weight, mean $\pm \mathrm{SD} \mathrm{kg} *$ & $65.33 \pm 15.32$ & $73.61 \pm 10.02$ & $69.00 \pm 12.85$ \\
Height median (95\% CI) $\mathrm{m}^{\dagger}$ & $1.68(1.58-1.71)$ & $1.60(1.57-1.66)$ & .15 \\
BMI, mean $\pm \mathrm{SD} \mathrm{kg} / \mathrm{m}^{2 *}$ & $23.99 \pm 4.60$ & $28.24 \pm 4.04$ & .28 \\
& & $.4 .56-1.67)$ & $.07 .21 \pm 4.64$
\end{tabular}

* One-way analysis of variance with Tukey honest significant difference test.

$\dagger$ Kruskal-Wallis with Dunn test.

BMI $=$ body mass index

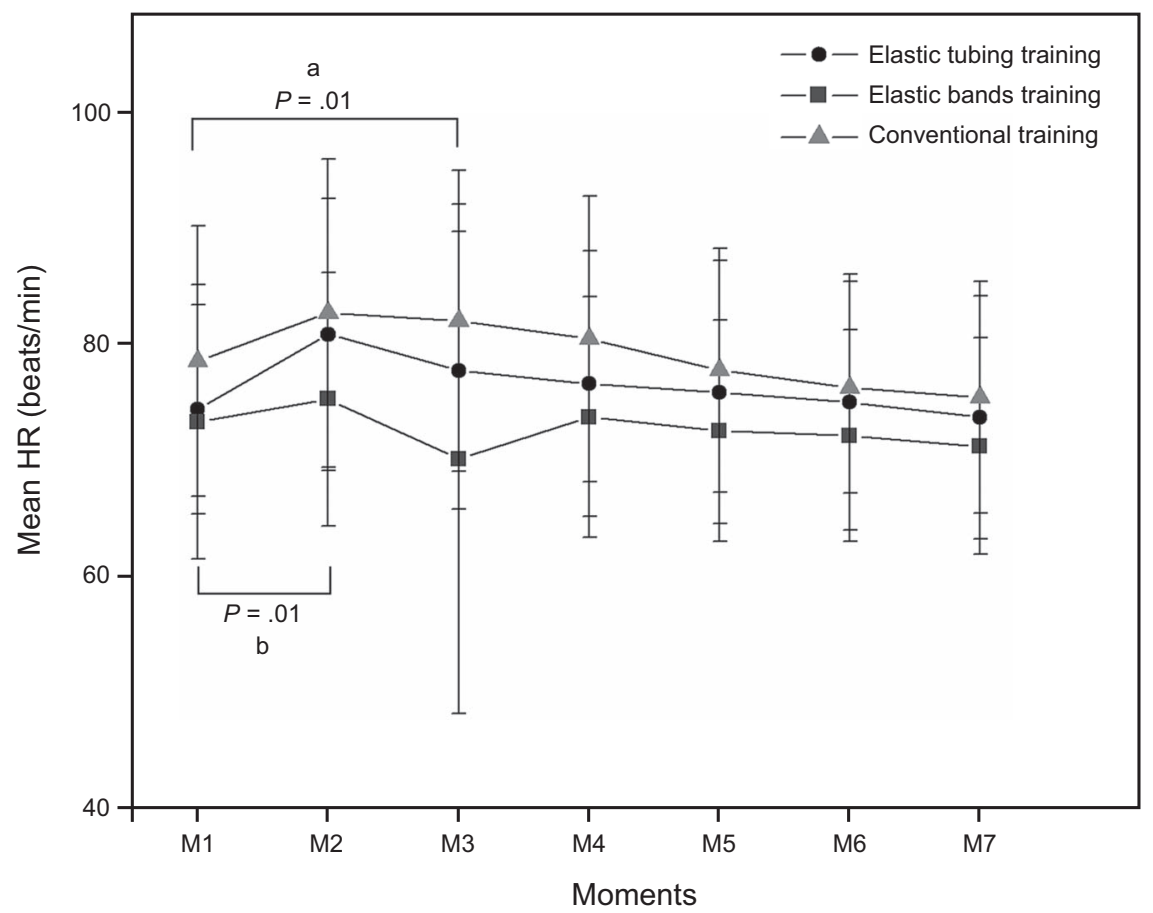

Fig. 2. Mean \pm SD Mean HR index in the elastic tubes, elastic bands, and conventional training groups in the rest and recovery conditions. $M 1=$ final 5 min of rest; M2 = 5 min initial recovery; M3 = 5th-10th min of recovery; M4 = 15th-20th min of recovery; M5 = 25th-30th min of the recovery; M6 = 40th-45th min of recovery; M7 = 55th-60th min of recovery. Letters denote the comparison between a = moments only in the tubes training and $b=$ moments in the elastic tubes training and elastic bands training.

a 2-tailed hypothesis test. The value obtained from the sample calculation was 10 subjects per group.

\section{Results}

The 3 groups analyzed did not present statistical differences in relation to the variables age, weight, height, or body mass index; therefore, they were considered homogeneous (Table 1). For the heart rate variability indices during recovery, the 2-way ANOVA analyzes showed that, during recovery, there was a moment effect for mean RR, rMSSD and HF $\left[\mathrm{ms}^{2}\right](P=.01)$. There was no effect among the groups for any index analyzed (Mean HR, $P=$ .37 ; SDNN, $P=.38$; rMSSD,$P=.18$; LF [nu], $P=.97$;
$\mathrm{HF}[\mathrm{nu}], P=.97 ; \mathrm{LF}\left[\mathrm{ms}^{2}\right], P=.43 ; \mathrm{HF}\left[\mathrm{ms}^{2}\right], P=.13$ $\mathrm{LF} / \mathrm{HF}$ ratio, $P=.95$ ), in addition to which there were no moment or group interactions (Mean HR, $P=.22$; SDNN, $P=.88 ; \mathrm{rMSSD}, P=.45 ; \mathrm{LF}[\mathrm{nu}], P=.27$; HF $[\mathrm{nu}]$, $P=.28$; LF $\left[\mathrm{ms}^{2}\right], P=.66$; HF $\left[\mathrm{ms}^{2}\right], P=.36$; and LF/HF ratio, $P=.46$ ).

The results of the mean HR variable in the rest and recovery conditions in the 3 groups studied are presented in Figure 2. For the elastic tubes group, there was a statistical difference at M2 in comparison with M1. For the elastic bands group, M2 and M3 presented significant differences in relation to M1. The conventional group did not present significant statistical differences between moments. 

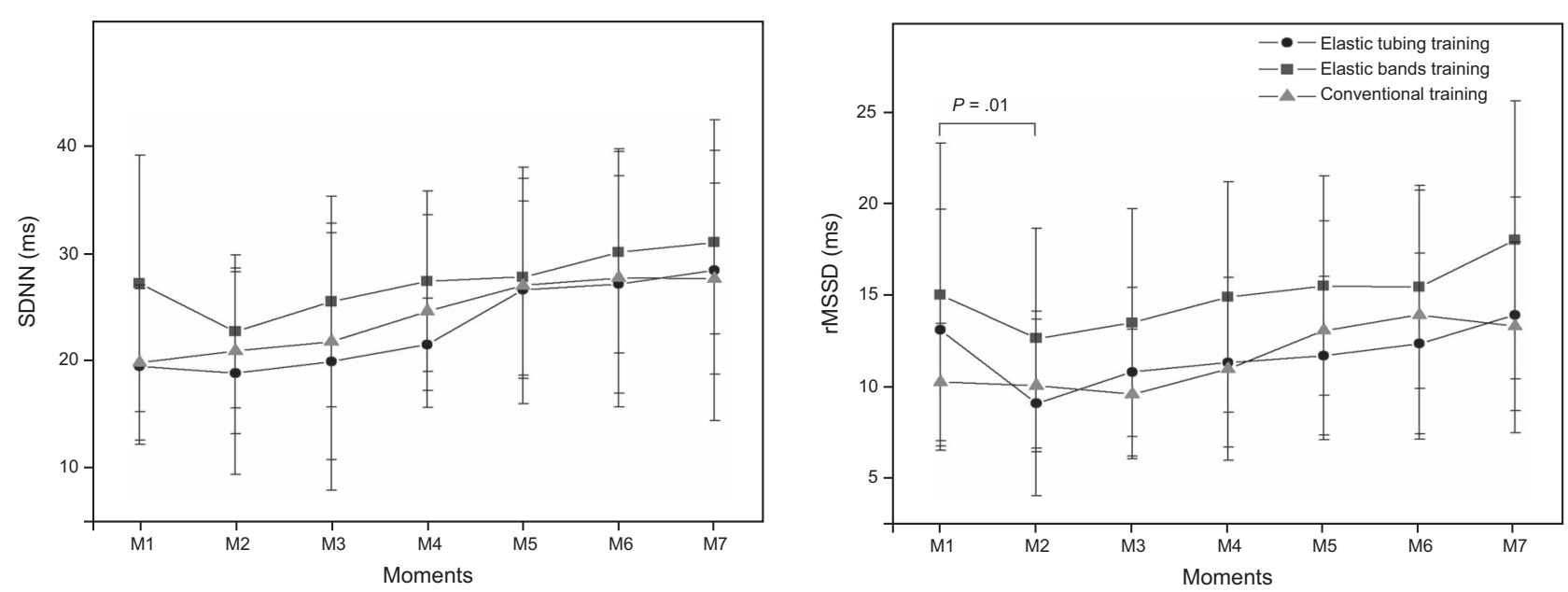

Fig. 3. Mean \pm SD of the SDNN and rMSSD indices in the elastic tubes, elastic bands, and conventional training groups in the rest and recovery conditions. $M 1=$ final 5 min of rest; M2 = 5 min initial recovery; M3 $=5$ th- 10 th min of recovery; M4 = 15th-20th min of recovery; M5 = 25th-30th $\mathrm{min}$ of recovery; M6 $=40$ th-45th $\mathrm{min}$ of recovery; M7 $=55$ th-60th $\min$ of recovery. $P$ value for comparison between moments only in the tubes training.

The results of the statistical indices of heart rate variability analyzed in the time domain under the rest and recovery conditions in the 3 groups studied are presented in Figure 3. The SDNN index did not present statistically significant differences between moments for the groups analyzed. For the rMSSD index, which represents parasympathetic modulation, the elastic tube group presented a statistically significant difference at M2 when compared with M1.

The results of heart rate variability analyzed in the frequency domain under the rest and recovery conditions in the 3 groups studied are presented in Figure 4. For the LF [nu], HF [nu], LF $\left[\mathrm{ms}^{2}\right]$, and LF/HF ratio, no statistical differences were observed among the moments for the 3 groups analyzed. For the HF $\left[\mathrm{ms}^{2}\right]$ index, representative of parasympathetic modulation, the elastic tubes group presented a statistical difference at M2 when compared with M1.

\section{Discussion}

The present study aimed to evaluate the effects of different types of resistance training (conventional vs elastic tubes vs elastic bands) in post-exercise recovery, single session on cardiac autonomic modulation in subjects with COPD. The results showed that heart rate increased after a resistance exercise in subjects with COPD; however, it recovered quickly. There was minimal change in heart-rate variability during recovery, with little difference between exercise strategies and heart-rate recovery and variability. There were minimum changes with the elastic tubes, whereas subjects had no differences in heart rate or autonomic modulation in the elastic-bands or conventional groups.
The pertinent literature addresses the chronic effects of resistance training in subjects with COPD; however, there is a lack of evidence to demonstrate the behavior after a single session of this type of training on cardiac autonomic modulation. This highlights the importance of the present study and characterizes it as the first study to our knowledge to verify the influence of different types of therapeutic resources against a single session of resistance exercise.

Although there are studies that describe the behavior of heart rate variability after aerobic exercise, ${ }^{25-28}$ few describe the autonomic response after resistance exercise. The studies that analyzed heart rate variability in the recovery period after resistance exercise observed increases in sympathetic modulation and reductions in parasympathetic modulation. ${ }^{29,30}$ However, previous studies do not compare post-exercise recovery in subjects with COPD using different therapeutic resources for resistance training. In addition, this literature does not present data showing the exact moment of autonomic nervous system recovery, considering a long recovery period.

It is known that, during the performance of resistance exercise, sympathetic activation occurs to supply the metabolic and cardiorespiratory demands required by the effort. ${ }^{31}$ This is a hypothesis that may explain the findings in the mean HR, in which an increase in values in the recovery period was observed for a maximum of $10 \mathrm{~min}$, which thus indicates sympathetic predominance due to exercise. ${ }^{31}$

During the recovery period, the heart rate gradually returns to basal values, mainly due to parasympathetic reactivation. ${ }^{31,32}$ The end of exercise promotes a loss of central control and cessation of baroreflex activation as well as other mechanisms that contribute significantly to increased parasympathetic modulation. ${ }^{32,33}$ This aspect can be observed by the behavior of the rMSSD index, which 

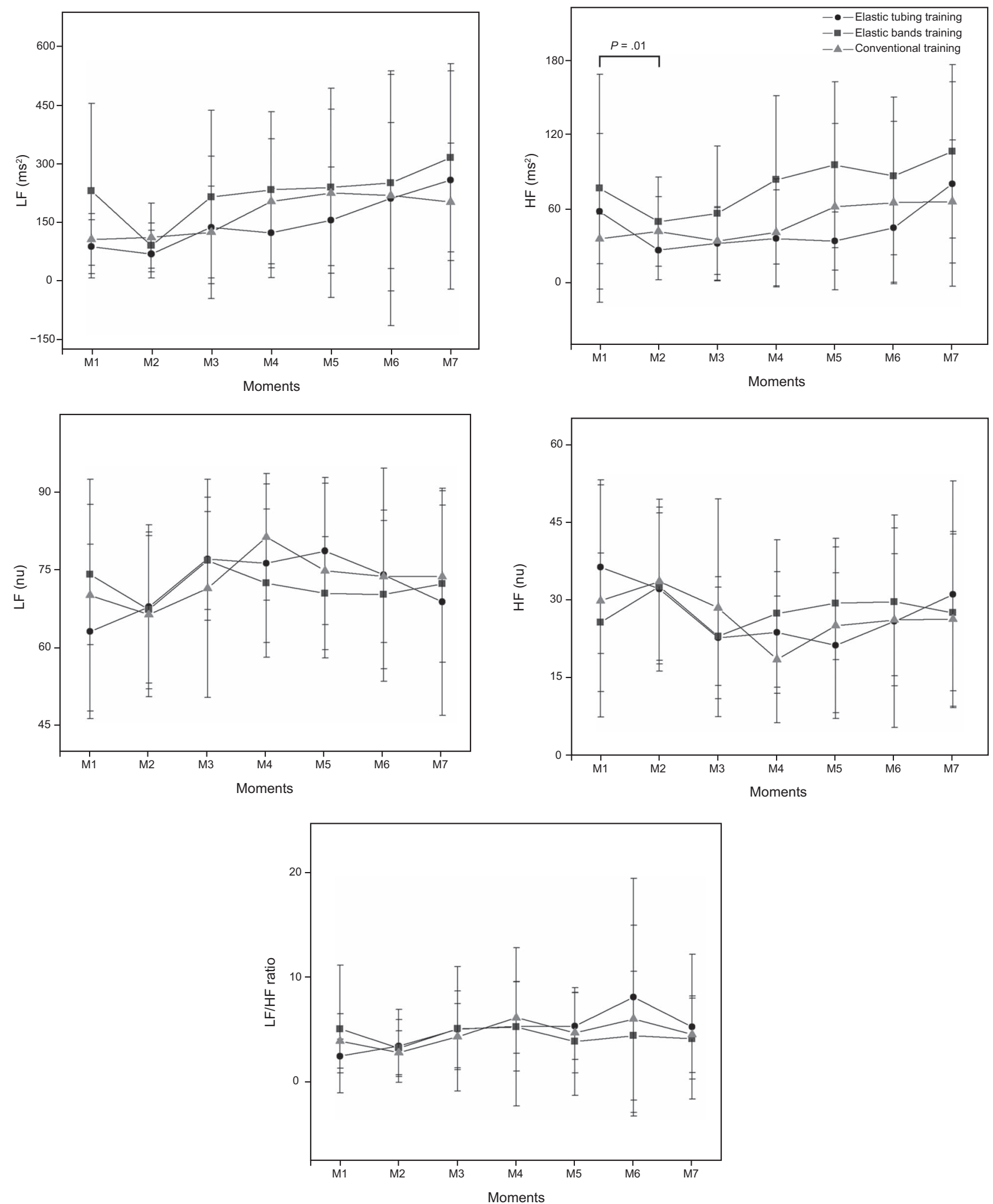

Fig. 4. Mean $\pm \mathrm{SD}$ of the LF (nu), HF (nu), LF $\left(\mathrm{ms}^{2}\right), \mathrm{HF}\left(\mathrm{ms}^{2}\right)$, and LF/HF ratio in the elastic tubes, elastic bands, and conventional training groups in the rest and recovery conditions. $M 1=$ final $5 \mathrm{~min}$ of rest; $\mathrm{M} 2=5 \mathrm{~min}$ initial recovery; $\mathrm{M} 3=5$ th- 10 th $\mathrm{min}$ of recovery; $\mathrm{M} 4=$ 15th-20th min of recovery; M5 = 25th-30th $\min$ of recovery; M6 = 40th-45th min of recovery; M7 $=55$ th-60th min of recovery. $P$ value for comparison between moments only in the tubes training. 


\section{Post-Exercise Recovery from Heart Rate Variability in COPD}

reflects parasympathetic modulation, which, at M2, corresponding to the initial 5 min of recovery, demonstrated a decrease in the values in relation to rest. These findings indicate sympathetic modulation caused acutely by resistance training, as these values approached baseline conditions (M1) at other moments, demonstrating a reactivation of parasympathetic modulation.

With regard to parasympathetic regulation after resistance exercise, Heffernan et al ${ }^{29}$ showed that 25 min after the end of the exercise, the HF index, which reflects parasympathetic modulation, ${ }^{13}$ was reduced when compared with aerobic exercise performed at $65 \% \dot{\mathrm{V}}_{\mathrm{O}_{2}}$ peak. These results may point to relative safety with respect to possible cardiovascular events when performing resistance exercise compared with maximum aerobic exercise. ${ }^{29}$ Oliveira et al ${ }^{34}$ also found a reduction in parasympathetic modulation after an exercise session in healthy men undergoing resistance training for 12 weeks when compared with maximum aerobic exercise, which once again reinforces the importance of evaluating cardiac autonomic modulation during the recovery period.

Mohammed et $\mathrm{al}^{35}$ verified, in a systematic review, that there is strong evidence to support the depression of baroreflex sensitivity in subjects with COPD. Possible contributors to this depression include alterations in intrathoracic pressure, hypoxia, and oxidative stress, which are common in patients with COPD. The baroreflex mechanism plays an important role in cardiovascular function, especially in the short-term regulation of blood pressure and heart rate. ${ }^{35}$ However, in our study, the impaired condition of COPD subject did not appear to influence post-exercise recovery following resistance training with different therapeutic resources, although further studies are necessary to prove this hypothesis.

The elastic tubes group was the only training group that presented statistical differences at M2 in relation to M1. Elastic resistance promotes greater physical demand required by the therapeutic resource because it provides a linear increase in tension from the beginning to the end of the contraction during the movement. ${ }^{7,8}$ This results are not unfavorable to the use of elastic tubes, as at M3 (5-10 $\min$ ) these subjects had already recovered. In other words, subjects had reached resting baseline values at $5 \mathrm{~min}$ after cessation of the single session, demonstrating that they were prepared for a new stimulus from an autonomic point of view. In addition, studies show that the gain in muscular strength with elastic tubes is similar to conventional bodybuilding and, therefore, in view of the cost/benefit, elastic tubes represent a resource to be prescribed for resistance exercises. ${ }^{8}$

The SDNN index, which reflects global variability, demonstrated an increase in the recovery moments for all the groups, which indicated an increase in the time of variation of the RR intervals due to post-exercise vagal reacti- vation; however, no statistical difference was observed between moments and groups. Lima et al ${ }^{30}$ carried out a study to verify the effects of strength exercise on heart rate variability in young people and showed that, after resistance training at $70 \%$ of one maximum repetition, the global variability presented an increase during the recovery period compared with the pre-exercise moment.

For the HF index, which also reflects parasympathetic modulation, for the elastic tubes group, there was a difference at M2 in relation to M1 similar to rMSSD index. Javorka et $\mathrm{l}^{32}$ evaluated heart rate variability in healthy men after a functional capacity test and observed reductions in the HF index during exercise and a progressive increase during recovery, which indicated parasympathetic reactivation in the spectral indices. These researchers also verified an increase in the LF index during recovery and indicated that this index is directly influenced by modifications in vagal modulation that promote fluctuations in the spectral bands or are indirectly influenced by changes in the sensitivity of the baroreflex..$^{32}$

Nicolino et $\mathrm{al}^{36}$ evaluated cardiac autonomic modulation after an acute resistance exercise session at different intensities of one maximum repetition in subjects with COPD. The HF and LF indices were higher at all recovery moments compared with the other indices in the training intensities of $60 \%$ and $90 \%$ of one maximum repetition. In addition, there was no statistical difference between the training protocols, which showed that the recovery of autonomic modulation in this population was similar regardless of the exercise intensity.

Karavirta et $\mathrm{al}^{37}$ found no effects of 21 weeks of resistance training on frequency-domain measures in healthy men age 40-67 y after twice weekly resistance training that consisted of 7-10 exercises. Training started with 3 sets of 15-30 repetitions at $40-60 \%$ of one maximum repetition and ended with 3 sets of 5-8 repetitions at 70$85 \%$ of one maximum repetition. Similarly, Wanderley et al ${ }^{38}$ demonstrated no change in HF after 8 months of resistance training in older adults with an age of $68 \pm 5 \mathrm{y}$, whether they presented uncontrolled hypertension or not.

Again, it should be emphasized that therapeutic resources with elastic resistance offer greater resistance at the end of the movement ${ }^{10}$; are highly versatile, which allows the patient to perform the exercise to its full range; ${ }^{7}$ are more practical because they require minimum space for training; and can be performed at home with a large number of movement arches, including both concentric and eccentric contractions. ${ }^{39,40}$ Thus, elastic tubes, despite modifying the rMSSD and HF $\left[\mathrm{ms}^{2}\right]$ indices, were shown to be a more practical and low-cost method when compared with elastic bands and conventional bodybuilding apparatus. ${ }^{8}$

As in the present study, Nicolino et $\mathrm{a}^{36}$ did not observe statistical differences in the $\mathrm{LF} / \mathrm{HF}$ ratio during the recovery period after resistance exercise. It is believed that the 


\section{Post-Exercise Recovery from Heart Rate Variability in COPD}

autonomic nervous system was able to act efficiently to restore and maintain the body's homeostasis in the face of physical effort. Such results may demonstrate the ability of patients with COPD to tolerate resistance training performed with different therapeutic resources.

Resistance training has been recommended to improve the autonomic function of patients with COPD. ${ }^{24,41}$ Santos et $\mathrm{al}^{24}$ investigated the influence of resistance training on cardiac autonomic modulation by using heart rate variability geometric methods in subjects with COPD. After 2 months of training, which ranged from 60 to $80 \%$ of one maximum repetition, the researchers observed a significant increase in the indices that reflect vagal modulation and global variability, and high beat-to-beat dispersion, which indicated an improvement in cardiac autonomic modulation as well as improved peripheral muscle strength. ${ }^{24}$

Ricci-Vitor et $\mathrm{al}^{41}$ found that resistance training was able to positively influence cardiac autonomic modulation of subjects with COPD when evaluated by the temporal and spectral indices of heart rate variability. However, it is again worth emphasizing that these studies ${ }^{24,41}$ show the chronic effects of training, whereas the present study demonstrated the acute results of cardiac autonomic after a session of resistance exercise.

As a limitation of the study, we pointed out the small sample size due to the specific geographic location and losses during the data collection process and analysis of heart rate variability. In addition, we should mention the influence of breathing frequency because it was not controlled as well and the lack of stratification of subjects according to severity of COPD.

It is important to study the heart rate variability indices in post-exercise recovery because an increase in sympathetic modulation in patients with COPD is associated with a higher rate of morbidity and mortality. ${ }^{42}$ In addition, low levels of parasympathetic reactivation in the recovery period can be considered an important risk factor for cardiac mortality. ${ }^{43}$ From an autonomic point of view, it can be inferred that the protocols proposed in this study can be safely applied because reactivation of the autonomic nervous system in the recovery period occurred in an efficient way.

\section{Conclusions}

The elastic tubes group, after single resistance training, promoted a minimum alteration in the post-exercise recovery of cardiac autonomic modulation in subjects with COPD, characterized by a decrease in the parasympathetic modulation immediately after exercise; however, at $5 \mathrm{~min}$ after exercise, the subjects with COPD had already recovered. Therefore, when considering its low cost and versatility, elastic tubes are a therapeutic resource option for resistance training in subjects with COPD.

\section{REFERENCES}

1. Sociedade Brasileira de Pneumologia e Tisiologia. Caracterização da doença pulmonar obstrutiva crônica (DPOC) - denifição, epidemiologia, diagnóstico e estadiamento. J Bras Pneumol 2004;30(5):10-14.

2. Global Initiative for Chronic Obstructive Lung Disease. Global Strategy for the Diagnosis, Management, and Prevention of COPD, Global Initiative for Chronic Obstructive Lung Disease (GOLD) 2017. www. goldcopd.org. Accessed November, 3, 2017.

3. Sala E, Roca J, Marrades RM, Alonso J, Gonzalez De Suso JM, et al. Effects of endurance training on skeletal muscle bioenergetics in chronic obstructive pulmonary disease. Am J Respir Crit Care Med 1999;159(6): 1726-1734.

4. Ries AL, Kaplan RM, Limberg TM, Prewitt LM. Effects of pulmonary rehabilitation on physiologic and psychosocial outcomes in patients with chronic obstructive pulmonary disease. Ann Intern Med 1995; 122(11):823-832.

5. Aliverti A, Macklem PT. The major limitation to exercise performance in COPD is inadequate energy supply to the respiratory and locomotor muscles. J Appl Physiol 2008;105(2):749-751; discussion 755-757.

6. du Moulin M, Taube K, Wegscheider K, Behnke M, van den Bussche $\mathrm{H}$. Home-based exercise training as maintenance after outpatient pulmonary rehabilitation. Respiration 2009;77(2):139-145.

7. Melchiorri G, Rainoldi A. Muscle fatigue induced by two different resistances: Elastic tubing versus weight machines. J Electromyogr Kinesiol 2011;21(6):954-959.

8. Ramos EM, de Toledo-Arruda AC, Fosco LC, Bonfim R, Bertolini GN, Guarnier FA, et al. The effects of elastic tubing-based resistance training compared with conventional resistance training in patients with moderate chronic obstructive pulmonary disease: a randomized clinical trial. Clin Rehabil 2014;28(11):1096-1106.

9. Babu AS, Balthillaya GM, Navada R, Kurien A. Theraband exercises with music for persons with haemophilia. Haemophilia 2013; 19(6):e359-e360.

10. Patterson RM, Stegink Jansen CW, Hogan HA, Nassif MD. Material properties of Thera-Band Tubing. Phys Ther 2001;81(8):1437-1445.

11. Paschoal MA, Petrelluzzi KF, Gonçalves NV. Study of heart rate variability in patients with chronic obstructive pulmonary disease. Rev Ciênc Med 2002;11(1):27-37.

12. Heart rate variability standards of measurement, physiological interpretation and clinical use. Circulation 1996;93(5):1043-1065.

13. Vanderlei LC, Pastre CM, Hoshi RA, Carvalho TD, Godoy MF. Basic notions of heart rate variability and its clinical applicability. Rev Bras Cir Cardiovasc 2009;24(2):205-217.

14. Stewart RI, Lewis CM. Cardiac output during exercise in patients with COPD. Chest 1986;89(2):199-205.

15. Stein PK, Nelson P, Rottman JN, Howard D, Ward SM, Kleiger RE. Heart rate variability reflects severity of COPD in PiZ alpha1-antitrypsin deficiency. Chest 1998;113(2):327-333.

16. Glaister M. Multiple sprint work: physiological responses, mechanisms of fatigue and the influence of aerobic fitness. Sports Med 2005;35(9):757-777.

17. Martinmäki K, Rusko H. Time-frequency analysis of heart rate variability during immediate recovery from low and high intensity exercise. Eur J Appl Physiol 2008;102(3):353-360.

18. Lohman TG, Roche AF, Martorell R. Anthropometric Standardization Reference Manual. Champaign, IL: Human Kinetics Books, 1988.

19. Standardization of Spirometry, 1994 Update. Am J Respir Crit Care Med 1995;152:1107-1136.

20. Vanderlei FM, Moreno IL, Vanderlei LC, Pastre CM, de Abreu LC, Ferreira C. Comparison of the effects of hydration with water or isotonic solution on the recovery of cardiac autonomic modulation. Int J Sport Nutr Exerc Metab 2015;25(2):145-153. 


\section{Post-Exercise Recovery from Heart Rate Variability in COPD}

21. Moreno IL, Vanderlei LC, Vanderlei FM, Pastre CM, Abreu LC, Ferreira C. Effects of water ingestion throughout exercise and recovery on cardiac autonomic modulation during and after exercise. Motriz 2016;22(3):174-182.

22. Godoy MF, Takakura IT, Correa PR. The relevance of nonlinear dynamic analysis (Chaos Theory) to predict morbidity and mortality in patients undergoing surgical myocardial revascularization. Arq Ciênc Saúde 2005;12(4):167-171.

23. Tarvainen MP, Niskanen JP, Lipponen JA, Ranta-Aho PO, Karjalainen PA. Kubios HRV - heart rate variability analysis software. Comput Methods Programs Biomed 2014;113(1):210-220.

24. Santos AA, Ricci-Vitor AL, Bragatto VS, Santos AP, Ramos EM, Vanderlei LC. Can geometric indices of heart rate variability predict improvement in autonomic modulation after resistance training in chronic obstructive pulmonary disease? Clin Physiol Funct Imaging 2017;37(2):124-130.

25. Nunan D, Jakovljevic DG, Donovan G, Singleton LD, Sandercock GR, Brodie DA. Resting autonomic modulations and the heart rate response to exercise. Clin Auton Res 2010;20(4):213-221.

26. Chen JY, Lee YL, Tsai WC, Lee CH Chen P, Li Y, et al. Cardiac autonomic functions derived from short term heart rate variability recordings associated with heart rate recovery after treadmill exercise test in young individuals. Heart Vessels 2011;26(3):282-288.

27. Casonatto J, Tinucci T, Dourado AC, Polito M. Cardiovascular and autonomic responses after exercise sessions with different intensities and durations. Clinics (Sao Paulo) 2011;66(3):453-458.

28. Kaikkonen P, Hynynen E, Mann T, Rusko H, Nummela A. Heart rate variability is related to training load variables in interval running exercises. Eur J Appl Physiol 2012;112(3):829-838.

29. Heffernan KS, Kelly EE, Collier SR, Fernhall B. Cardiac autonomic modulation during recovery from acute endurance versus resistance exercise. Eur J Cardiovasc Prev Rehabil 2006;13(1):80-86.

30. Lima AH, Forjaz CL, Silva GQ, Menêses AL, Silva AJ, Ritti-Dias RM. Acute effect of resistance exercise intensity in cardiac autonomic modulation after exercise. Arq Bras Cardiol 2011;96(6):498-503.

31. Esco MR, Olson MS, Williford HN, Blessing DL, Shannon D, Grandjean $P$. The relationship between resting heart rate variability and heart rate recovery. Clin Auton Res 2010;20(1):33-38.

32. Javorka M, Zila I, Balhárek T, Javorka K. Heart rate recovery after exercise: relations to heart rate variability and complexity. Braz J Med Biol Res 2002;35(8):991-1000.
33. O'Leary DS. Autonomic mechanisms of muscle metaboreflex control of heart rate. J Appl Physiol 1993;74(4):1748-1754.

34. Oliveira RS, Costa MV, Pedro RE, Polito MD, Avelar A, Cyrino ES, et al. Acute cardiac auto-nomic responses after a bout of resistance exercise. Sci Sports 2012;27(6):357-364.

35. Mohammed J, Meeus M, Derom E, Da Silva H, Calders P. Evidence for autonomic function and its influencing factors in subjects with COPD: A systematic review. Respir Care 2015;60(12): 1841-1851.

36. Nicolino J, Ramos D, Leite MR, Rodrigues FM, de Alencar Silva BS, Tacao GY, et al. Analysis of autonomic modulation after an acute session of resistance exercise at different intensities in chronic obstructive pulmonary disease patients. Int J Chron Obstruct Pulmon Dis 2015;29:10:223-229.

37. Karavirta L, Tulppo MP, Laaksonen DE, Nyman K, Laukkanen RT, Kinnunen $\mathrm{H}$, et al. Heart rate dynamics after combined endurance and strength training in older men. Med Sci Sports Exerc 2009;41: 1436-1443.

38. Wanderley FA, Moreira A, Sokhatska O, Palmares C, Moreira P, Sandercock G, et al. Differential responses of adiposity, inflammation and autonomic function to aerobic versus resistance training in older adults. Exp Gerontol 2013;48:326-333.

39. Colado JC, Triplett NT. Effects of a short-term resistance program using elastic bands versus weight machines for sedentary middleaged women. J Strength Cond Res 2008;22(5):1441-1448.

40. Santos GM, Tavares GM, Gasperi G, Bau GR. Mechanical evaluation of the resistance of elastic bands. Rev Bras Fisioter 2009;13(6): 521-526.

41. Ricci-Vitor AL, Bonfim R, Fosco LC, Bertolini GN, Ramos EM, Ramos D, et al. Influence of the resistance training on heart rate variability, functional capacity and muscle strength in the chronic obstructive pulmonary disease. Eur J Phys Rehabil Med 2013;49(6): 793-801.

42. Andreas S, Haarmann H, Klarner S, Hasenfuss G, Raupach T. Increased sympathetic nerve activity in COPD is associated with morbidity and mortality. Lung 2014;192(2):235-241.

43. Albert CM, Mittleman MA, Chae CU, Lee IM, Hennekens CH, Manson JE. Triggering of sudden death from cardiac causes by vigorous exertion. N Engl J Med 2000;343(19):1355-1361. 